\title{
Apuntes sobre la importancia ecológica, ambiental y social de la arenca Triportheus magdalenae (Steindachner, 1878). Un ejemplo de endemismo invisibilizado
}

\section{Notes about ecological, environmental and social importance of the arenca Triportheus magdalenae. An example of invisibilized endemism}

\author{
Juan Carlos Valdelamar-Villegas
}

*Autor de correspondencia: jvaldelamarv1@unicartagena.edu.co

Recibido: 26 de abril de 2018 - Aceptado: 16 de octubre de 2018

1 Grupo de Química Ambiental y Computacional, Universidad de Cartagena, Campus Zaragocilla, Cartagena, Colombia

Palabras clave:

ecología trófica; endémica; recurso pesquero; reproducción ecology; endemic; fishing resources; reproduction

\section{Resumen}

La arenca (Triportheus magdalenae) es una especie endémica de Colombia que habita principalmente la cuenca del río Magdalena-Cauca. En la presente revisión se integra la información de los pocos reportes científicos en los que se ha incluido a la especie, con el propósito de resaltar su importancia ecológica, ambiental y social. Los resultados revelaron que T. magdalenae habita principalmente en humedales de agua dulce con bajos valores de dióxido de carbono y acidez moderada. La especie juega un papel importante en el entramado trófico de los ecosistemas acuáticos como regulador del plancton, a partir del cual es posible que obtenga la energía y nutrientes necesarios para su ciclo de vida, que ocurre de forma asincrónica y multiporcional. Además de su importancia ecológica y ambiental, la arenca históricamente ha sido fuente de proteína para muchas poblaciones que habitan en la cuenca, y actualmente ha sido identificado el uso potencial de algunos de sus derivados para aplicaciones en la industria alimenticia y farmacéutica. Sin embargo, la actual presión pesquera y ambiental puede estar comprometiendo la viabilidad de su población en el futuro.

\section{Abstract}

The arenca (Triportheus magdalenae) is an endemic fish species of Colombia which mainly inhabits the Magdalena River basin. In this review we integrated information from the few scientific reports in which the species has been included, in order to identify its ecological, environmental, and social importance. The results of this evaluation revealed that T. magdalenae inhabits strictly in freshwater places with low values of carbon dioxide and moderate acidity. The species plays a key role in the aquatic ecosystem trophic network, such as a plankton regulator, from which it possibly obtains the required energy and nutrients to carry out its reproduction, which occurs asynchronously and multiportional. In addition to its environmental importance, the arenca has been historically recognized as a source of protein for many populations that inhabit the Magdalena River basin, and has now been identified as a potential source of some useful derivatives for applications in food and pharmaceutical industry. However, current fishing and environmental pressure may be compromising the viability of this resource in the future. 


\section{Introducción}

Triportheus magdalenae (figura 1), conocida vernáculamente como arenca, es una especie que se caracteriza por tener el cuerpo alargado y comprimido lateralmente. Posee una quilla en la parte media ventral y grandes aletas pectorales. La línea lateral está claramente definida, adopta una forma curva ventralmente y tiene 37 a 42 escamas (Dahl, 1971; Malabarba, 2004; JiménezSegura et al., 2011). La longitud total (Lt) de los individuos considerados como adultos varía de 16,0 a 17,0 cm en machos y de 19,5 a 21,7 cm en hembras; los machos alcanzan un peso total (Pt) promedio de $70,13 \mathrm{~g}$, mientras que en las hembras este valor puede ser de 94,14 g (Bolívar et al., 2006a). La especie presenta relaciones biométricas de tipo lineal entre la longitud estándar (Le) y la Lt, mientras que entre Le y Pt se ha identificado una relación de tipo exponencial (tabla 1).

La arenca pertenece a la familia Triportheidae y, dentro de ésta, al género Triportheus, donde según Mariguela et al. (2015) conforma un grupo hermano con T. culter (figura 1), del cual se separó hace aproximadamente 6,7 \pm 4,6 Millones de años. Así mismo, se ha identificado su origen en la cuenca del Amazonas durante la formación del sistema montañoso de los Andes septentrionales, a partir del Mioceno medio tardío (hace 12 millones de años) y el Plioceno temprano (hace 4,5 millones de años). El aislamiento experimentado durante los periodos posteriores facilitó el endemismo de la especie en la cuenca del río Magdalena en Colombia (Maldonado-Ocampo y Usma-Oviedo, 2006).

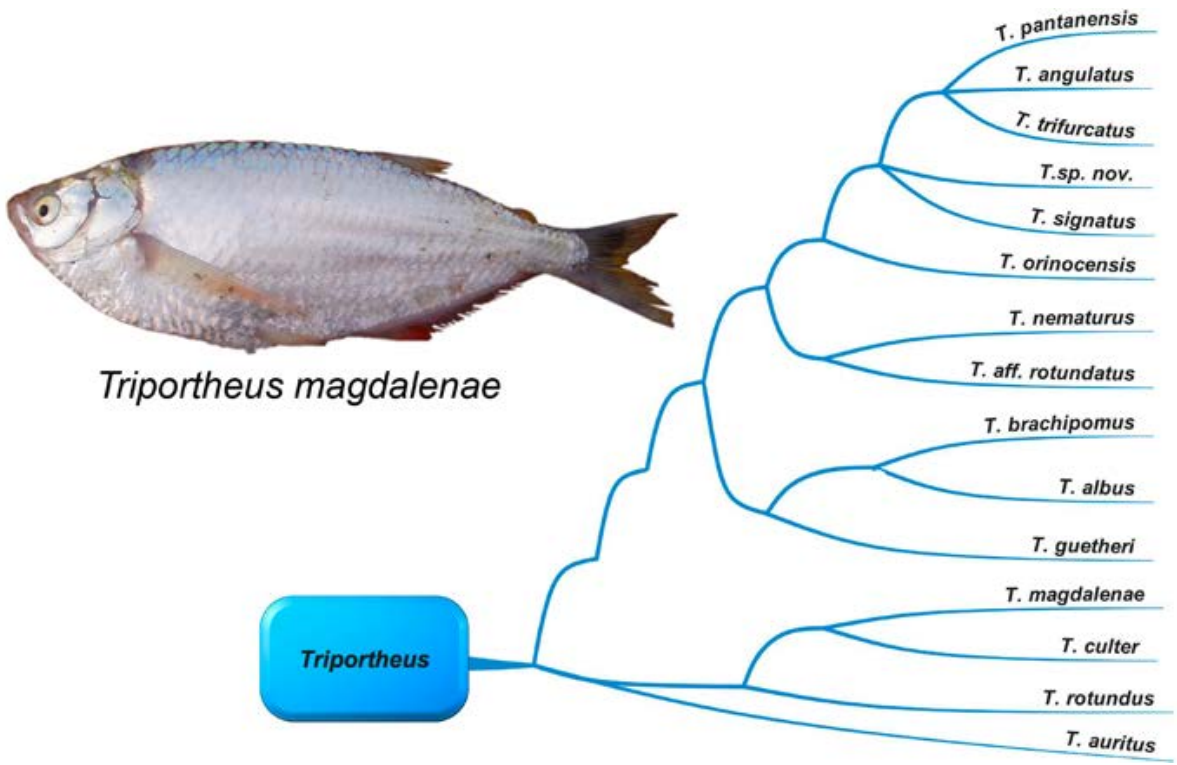

Figura 1. Relaciones filogenéticas entre T. magdalenae y otras especies del género Triportheus. Adaptado de Mariguela et al. (2015).

Tabla 1. Relaciones biométricas registradas para T. magdalenae. n: número total de individuos; H: hembras; M: machos; Lt: longitud total $(\mathrm{cm})$, Le: longitud estándar (cm); Pt: peso total (g); $\mathrm{r}^{2}$ : coeficiente de determinación.

\begin{tabular}{llrrl}
\hline Relación & Sexo & $\mathbf{n}$ & $\mathbf{r}^{2}$ & Autor \\
\hline $\mathrm{Lt}=17,5+1,17 \mathrm{Le}$ & - & 460 & - & Rodríguez y Rodríguez (1976) \\
$\mathrm{Pt}=0,005 \mathrm{Lt}^{3,52}$ & - & 460 & - & \\
$\mathrm{Lt}=\mathrm{Le}-1,58 / 0,76$ & $\mathrm{H}$ & 237 & 0,87 & Bolívar et al. (2006a) \\
$\mathrm{Lt}=\mathrm{Le}-0,96 / 0,79$ & $\mathrm{M}$ & 284 & 0,92 & \\
$\mathrm{Pt}=0,005 \mathrm{Lt}^{3,21}$ & $\mathrm{H}$ & 237 & 0,85 & \\
$\mathrm{Pt}=0,015 \mathrm{Lt}^{2,85}$ & $\mathrm{M}$ & 284 & 0,73 & \\
$\mathrm{Pt}=0,040 \mathrm{Lt}^{2,43}$ & - & 1498 & 0,73 & CCI $(2007)$ \\
\hline
\end{tabular}


Históricamente T. magdalenae ha jugado un papel importante en la dieta de los pobladores de la cuenca del río Magdalena. Los registros arqueológicos así lo demuestran, por lo menos desde el periodo Arcaico o formativo temprano, en el Conchero (sitio de organización de cazadores recolectores) de Rotinet, ubicado en el occidente del embalse del Guájaro, en el sur del departamento del Atlántico (Archila, 1993; Peña, 2013). Igualmente se ha encontrado evidencia de su uso por parte de las poblaciones que habitaban la cuenca media del río Magdalena, entre Honda-Tolima y Puerto Bogotá-Cundinamarca, en el periodo formativo tardío (Peña-León, 2011).

Además de la conocida importancia de T. magdalenae como fuente de proteína, algunos estudios sugieren que la especie también juega un papel importante en la regulación del zooplancton en los ecosistemas donde habita, función que probablemente contribuye con el control de los productores primarios de los ecosistemas donde suele habitar (Moreno 2005; Morales y García-Alzate, 2018).

A pesar de ser reconocida la importancia de la arenca, son escasos los estudios sobre este recurso. Por esta razón, se realizó la presente revisión donde se destacan algunos aspectos relacionados con su distribución geográfica, las características ambientales de los ecosistemas que habita, su ecología trófica y reproductiva, la importancia socioeconómica de la especie y algunos riesgos ambientales a los que ha estado sujeta durante los últimos años. Finalmente, se espera que los elementos destacados sirvan para visibilizar la importancia que reviste $T$. magdalenae para la conservación de la biodiversidad íctica en la cuenca del río Magdalena y la manera como ésta se puede ver afectada por las actividades antrópicas.

\section{Materiales y métodos}

Para el desarrollo de la presente revisión se consultaron trabajos de grado de algunas instituciones universitarias, de manera física y en línea. También se revisaron las bases de datos Sciencedirect, Pubmed y el buscador Google Scholar. Posteriormente, la información recopilada fue organizada de forma tabular y gráfica empleando el programa computacional Excel de Microsoft y el Paquete estadístico GrapPad Prism 6.0.

\section{Distribución geográfica}

La arenca se distribuye en la cuenca del río MagdalenaCauca (Villa-Navarro et al., 2003, 2006; Mojica et al., 2006; Gil-Calderón y Márquez, 2015), desde la parte alta del río La Miel, en el departamento de Caldas, hasta la desembocadura del río Magdalena, en el departamento del Atlántico, y en ecosistemas costeros como la Ciénaga Grande de Santa Marta, y la desembocadura del Canal del Dique, en la Bahía de Cartagena (figura 2), razón por la cual Polanía et al. (2001) consideran a la especie como estenohalina. A pesar de tener un amplio rango de distribución, debido a su carácter migratorio (López-Casas y Jiménez-Segura, 2015), la mayoría de reportes sobre la arenca se han realizado en los departamentos de Bolívar (32\%), Santander (16\%) y Magdalena (12\%).

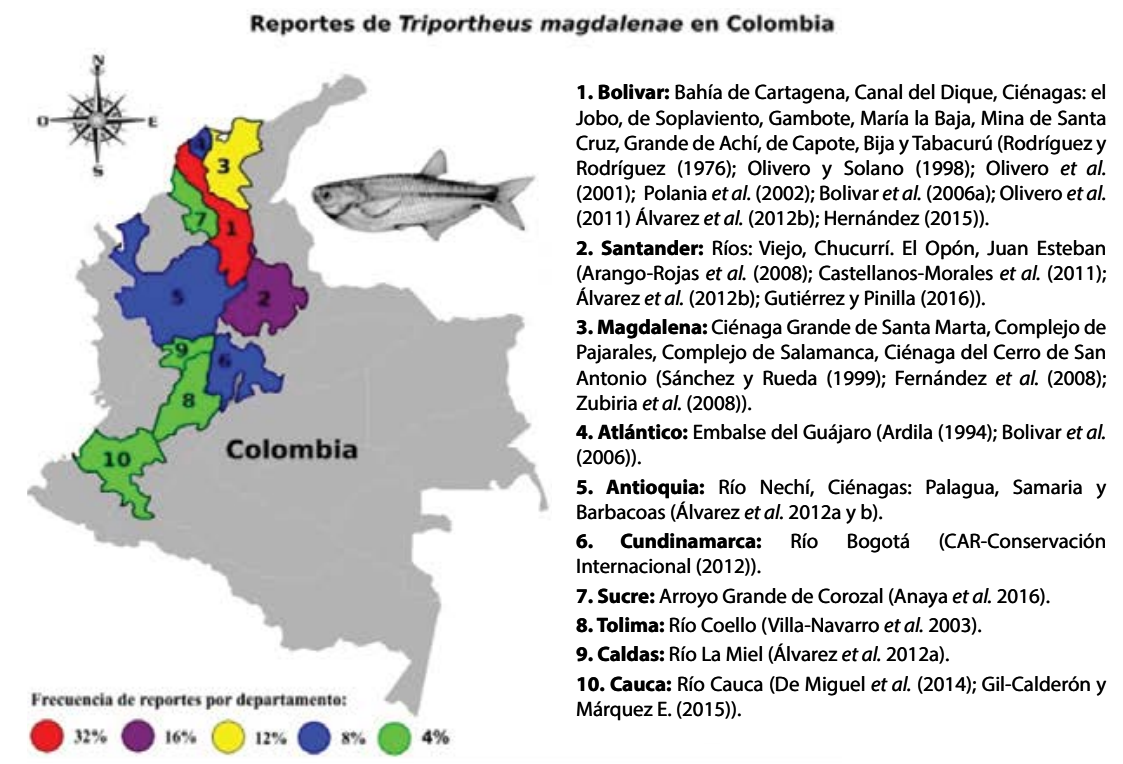

Figura 2. Área de distribución y frecuencia (\%) de reportes de T. magdalenae en Colombia. Elaborado a partir de Rodríguez y Rodríguez (1976). 
Aunque Zapata y Usma (2013) no incluyen a T. magdalenae en la lista de peces migratorios de Colombia, es bien conocido que la arenca migra desde la parte baja hasta la parte alta en la cuenca del río Magdalena, fenómeno conocido como "subienda", entre los meses de diciembre a febrero, coincidiendo con la época seca (CCI, 2007). Durante este período en las ciénagas disminuye la altura de la columna de agua, lo que facilita la agregación de los individuos de la especie y con ello su susceptibilidad a la acción de sus predadores, razón por la cual posiblemente éstos salen de los ecosistemas para protegerse e iniciar su proceso reproductivo (Gutierrez y Pinilla, 2016).

\section{Características fisicoquímicas de los humedales donde habita T. magdalenae}

Uno de los aspectos que más influye en la distribución de los peces es su capacidad para tolerar los cambios en las condiciones fisicoquímicos del agua, ya que de no poseer los mecanismos de adaptación apropiados, pueden experimentar alteraciones significativas en algunos de sus procesos bioquímicos vitales (Aazami et al., 2015), entre estos: desnaturalización de proteínas como las enzimas metabólicas cuando aumenta temperatura del agua (Lahnsteiner y Mansour, 2012) e interrupción del intercambio gaseoso cuando se reduce de manera significativa la concentración de oxígeno disuelto, o por cambios en la concentración de sales en el medio que afectan la osmolaridad de los organismos (Vargas-Chacoff et al., 2015).

Atendiendo a las anteriores consideraciones, en la tabla 2 se muestra el intervalo de variación de algunos parámetros fisicoquímicos del agua en los ecosistemas donde se ha reportado la presencia de T. magdalenae. Esta información permite afirmar que la especie vive en condiciones de oxígeno disuelto moderado y alto, con $\mathrm{pH}$ superior al neutro y temperatura superior a los $27{ }^{\circ} \mathrm{C}$, en cuerpos de agua cuya profundidad supera los $2 \mathrm{~m}$, con amplio rango de transparencia, dureza y salinidad, aunque la mayoría de reportes la ubica en humedales de agua dulce. Además, prefiere aguas con bajos valores de dióxido de carbono, silicatos y amonio. Puede tolerar acidez moderada y altos valores de sulfatos en el agua, así como también variaciones en la concentración de sólidos suspendidos.

Tabla 2. Intervalo de variación de algunos parámetros fisicoquímicos del agua para distintos lugares donde ha sido registrada $T$. magdalenae.

\begin{tabular}{|c|c|c|}
\hline Parámetro fisicoquímico & $\begin{array}{l}\text { Mínimo- } \\
\text { Máximo }\end{array}$ & Fuente \\
\hline Oxígeno disuelto (mg/L) & $3,3-7,9$ & $\begin{array}{l}\text { Rodríguez y Rodríguez (1976); Fernández et al. (2008); Benavidez y Valdelamar } \\
\text { (2010) }\end{array}$ \\
\hline $\mathrm{pH}$ & $7,13-9,52$ & $\begin{array}{l}\text { Rodríguez y Rodríguez (1976); Fernández et al. (2008); Benavidez y Valdelamar } \\
\text { (2010); Barrios y García-Alzate (2017) }\end{array}$ \\
\hline Temperatura $\left({ }^{\circ} \mathrm{C}\right)$ & $27,9-34,0$ & $\begin{array}{l}\text { Rodríguez y Rodríguez (1976); Fernández et al. (2008); Benavidez y Valdelamar } \\
\text { (2010); Barrios y García-Alzate (2017) }\end{array}$ \\
\hline Profundidad (m) & $2,55-5,17$ & Morales y García-Alzate (2018) \\
\hline Transparencia $(\mathrm{cm})$ & $5,0-180$ & $\begin{array}{l}\text { Rodríguez y Rodríguez (1976); Fernández et al. (2008); Benavidez y Valdelamar } \\
\text { (2010) }\end{array}$ \\
\hline Sólidos suspendidos (mg/L) & $8-210$ & Ortíz-Muñoz y Álvarez-León (2008); Barrios y García-Alzate (2017) \\
\hline Salinidad & $0-32$ & Sánchez y Rueda (1999) \\
\hline Conductividad $(\mu \mathrm{S} / \mathrm{cm})$ & $0,05-180$ & $\begin{array}{l}\text { Fernández et al. (2008); Ortíz-Muñoz y Álvarez-León (2008); Barrios y García- } \\
\text { Alzate (2017) }\end{array}$ \\
\hline Alcalinidad (mmol/L) & $11-242,85$ & $\begin{array}{l}\text { Fernández et al. (2008); Ortíz-Muñoz y Álvarez-León (2008); Benavidez y } \\
\text { Valdelamar (2010); Barrios y García-Alzate (2017) }\end{array}$ \\
\hline Dureza (mmol/L) & $3,58-213,2$ & $\begin{array}{l}\text { Rodríguez y Rodríguez (1976); Fernández et al. (2008); Ortíz-Muñoz y Álvarez- } \\
\text { León (2008); Benavidez y Valdelamar (2010) }\end{array}$ \\
\hline $\mathrm{CO}_{2}(\mathrm{ppm})$ & $2,27-5,31$ & Benavidez y Valdelamar (2010) \\
\hline Silicatos (mg/L) & $0-13,67$ & Fernández et al. (2008) \\
\hline Amonio (mg/L) & $0,47-2,90$ & Fernández et al. (2008); Barrios y García-Alzate (2017) \\
\hline Sulfatos (mg/L) & $35-51,1$ & Fernández et al. (2008); Barrios y García-Alzate (2017) \\
\hline Acidez (mmol/L) & $0,1-6,4$ & Fernández et al. (2008); Barrios y García-Alzate (2017) \\
\hline $\mathrm{DBO}(\mathrm{mg} / \mathrm{L})$ & $1-16$ & $\begin{array}{l}\text { Ortíz-Muñoz y Álvarez-León (2008); Fernández et al. (2008); Barrios y García- } \\
\text { Alzate (2017) }\end{array}$ \\
\hline
\end{tabular}


El conocimiento de las condiciones fisicoquímicas del agua toleradas por T. magdalenae es de gran importancia para poder inferir el impacto que tendrían los cambios de esos parámetros acuáticos sobre la viabilidad de la especie, principalmente en el actual contexto de cambio climático global. Igualmente, este tipo de información es fundamental para gestionar estrategias de manejo y conservación de la especie, así como en la planeación y ejecución de sistemas de producción acuícola naturales y artificiales.

\section{Reproducción de la arenca}

El análisis de las estrategias reproductivas de los peces constituye un aspecto fundamental para la implementación de planes de manejo pesquero que garanticen la sostenibilidad de este recurso. Este tipo de análisis incluye, entre otros aspectos, la caracterización morfológica de sus órganos reproductivos y los cambios que estos experimentan durante el proceso reproductivo (Nakamura, 2013).

Dentro de los aspectos relacionados con su reproducción y desarrollo, el género Triportehus posee un número diploide de 52 cromosomas, con predominancia de cromosomas meta y sub-metacéntricos, y mantienen el sistema de cromosomas sexuales ZZ/ZW (Marquioni et al., 2013). La arenca posee un par de gónadas que se ubican en la parte superior de la cavidad celómica, y se caracterizan por tener forma aplanada (Rodríguez y Rodríguez, 1976; Bolívar et al., 2006a); además, están cubiertas por una fina capa de tejido conectivo y en su interior se ubican las lamelas que contienen los ovocitos, en el caso de las hembras, y los lóbulos que contienen las células espermáticas, en los machos.

Las gónadas de la arenca, al igual que en el resto de especies, experimentan cambios macroscópicos e histológicos (tabla 3) durante el ciclo de desarrollo gonadal (Bolívar et al., 2006b), y están sincronizadas con la estacionalidad climática de la región para garantizar que sus larvas sobrevivan y se desarrollen durante el periodo que ofrece las mejores condiciones ambientales (OchoaOrrego et al., 2015). Previo a la migración y al inicio de la maduración gonadal, T. magdalenae acumula grasa perivisceral para garantizar la disponibilidad de nutrientes y energía durante su travesía (Bolívar et al., 2007).

Las anteriores estrategias reproductivas de T. magdalenae son favorecidas por la capacidad de la especie para realizar varios desoves de forma asincrónica, como se ha observado en los estudios de reproducción de la especie, basados en el análisis histológico de sus gónadas (Bolívar et al., 2006b), que han demostrado en sus ovarios la presencia de ovocitos en diferentes estados de desarrollo (figura 3). Esta estrategia reproductiva, a pesar de favorecer la rápida recuperación de su población, muy probablemente no será suficiente para compensar en el largo plazo la actual presión pesquera que se ejerce sobre esta especie y otros representantes de la ictiofauna, como el bocahico (Prochilodus magdalenae) y el bagre (Pseudoplatystoma magdaleniatum) en la cuenca del río Magdalena (Mojica, 2002; Alonso et al., 2014).

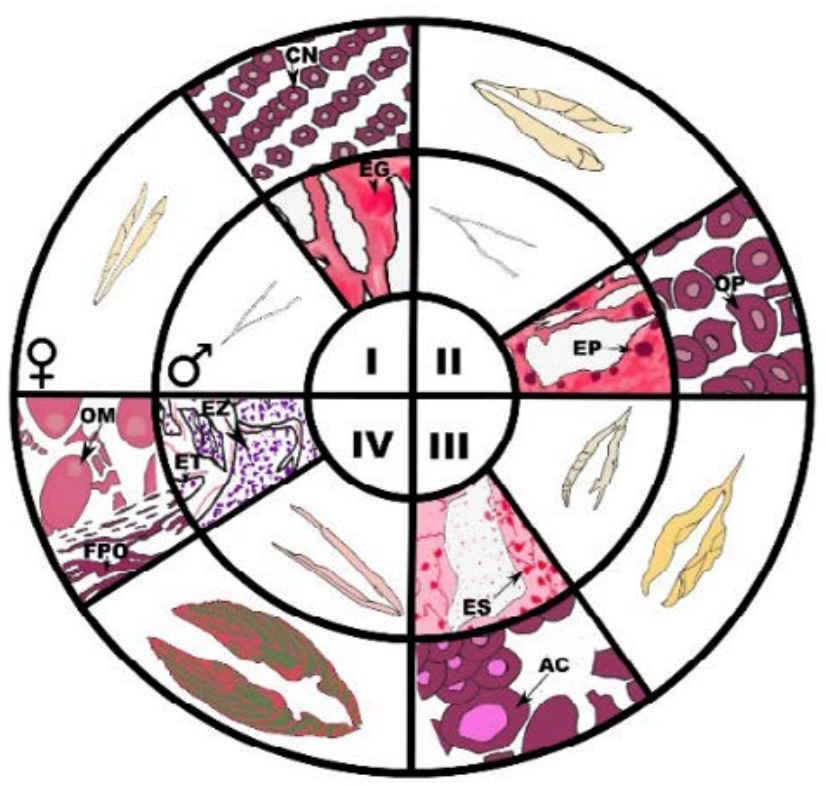

Figura 3. Estados de desarrollo gonadal macroscópico e histológico de T. magdalenae. CN: cromatina nucleólo, OP: ovocito perinucleolar, AC: alveólo cortical, OM: ovocito maduro, FPO: folículo post-ovulatorio, EG: espermatogónia, EP: Espermatocito primario, ES: espermatocito secundario, ET: espermátide, EZ: espermatozoide. Adaptado de Bolívar et al. (2006b). 
Tabla 3. Características macroscópicas e histológicas observadas durante el desarrollo gonadal de T. magdalenae. Fuente: Bolívar et al. (2006b).

\begin{tabular}{lll}
$\begin{array}{l}\text { Estado de } \\
\text { maduración }\end{array}$ & Ovarios & Testículos \\
\hline I & Macroscópico & $\begin{array}{l}\text { Ovarios pequeños, traslúcidos, con poca Pequeños, traslúcidos con pobre irrigación sanguínea. } \\
\text { irrigación sanguínea }\end{array}$
\end{tabular}

Histológico Mayor frecuencia de ovocitos basófilos Dominancia de espermatogónias, de forma poliédrica, con formas poliédricas y cuyo tamaño en caracterizadas por su gran tamaño, sin núcleo aparente y promedio es de $47,46 \pm 8,1 \mu \mathrm{m}$ coloración débilmente basófila.

II Macroscópico Pequeños de color rosado claro, planos Color blanco lechoso, todavía con pobre irrigación lateralmente, se aprecia mayor irrigación sanguínea.

sanguínea

Histológico Dominancia de ovocitos perinucleolares En esta fase son dominantes los espermatocitos primarios, de forma poliédrica, con núcleo central y caracterizados por ser más redondeados y adquirir cromatina densa. El diámetro promedio de coloración basófila.

$78,85 \pm 25,35 \mu \mathrm{m}$.

III Macroscópico Ovarios de color amarillo, de mayor tamaño Órganos de mayor tamaño que en el estado II y color que el estado II, y mayor irrigación sanguínea. blanco lechoso.

Histológico Estado denominado como alveolo cortical, Durante esta fase se observan con mayor frecuencia en el que son muy frecuentes los ovocitos espermatocitos secundarios de menor tamaño que los con coloración menos basófila y diámetro primarios, con coloración basófila.

promedio de 11,15 $\pm 2,80 \mu \mathrm{m}$. La capa de células foliculares es plana y toma una forma ondulada, lo que le confiere al ovocito una forma esférica e irregular, y la aparición de circunferencias de color blanco, conocidas como alvéolos corticales y cuerpos balbianos.

IV Macroscópico Ovarios de mayor tamaño, con abundante Testículos de mayor tamaño y color beige. irrigación sanguínea, coloración verde oliva, y abundantes ovocitos de color amarillo.

Histológico Durante esta fase inicia la vitelogénesis, los Se observan espermátidas con forma elíptica de color ovocitos se tornan eosinófilos, con diámetro basófilo, y espermatozoides con cabeza redondeada, con promedio de 256,67 \pm 48,64 $\mu \mathrm{m}$, y los coloración basófila, cola delgada de marcada coloración nucléolos aumentan en diámetro y número, y eosinófila, traslúcida en forma de filamento corto. se sitúan nuevamente en la periferia; también aparece el vitelo en forma de gránulos que se acumulan gradualmente en el citoplasma, hasta que los ovocitos alcanzan un diámetro promedio de 599,82 $\pm 99,8 \mu \mathrm{m}$. En esta fase también es posible observar folículos postovulatorios y atrésicos. 


\section{Ecología trófica}

El conocimiento de los hábitos alimenticios de las especies es un aspecto de gran importancia para comprender algunos elementos básicos de su historia de vida, la manera como estas interactúan con otros organismos del ecosistema que habitan (Smith et al., 2011) y las consecuencias que pueden tener las alteraciones ambientales sobre la composición y estructura de las comunidades que conforman su dieta (Motta et al., 2016).

En ese sentido, los conocimientos que se tienen sobre la arenca indican que es una especie estenofágica, con comportamiento orientado hacia el consumo de zooplancton, principalmente de microcrustáceos y, en menor medida, de restos vegetales, fitoplancton, insectos y moluscos (figura 4) (Moreno, 2005; Benavidez y Valdelamar, 2010; Morales y García-Alzate, 2018). Esta característica le confiere a la arenca un papel importante al ser una de las especies reguladoras de los eslabones inferiores de la red trófica (Holmlund y Hammer, 1999) en los ecosistemas acuáticos de la cuenca del río Magdalena.

\section{Importancia socioeconómica}

\section{Pesquería}

Históricamente T. magdalenae ha sido considerada como una especie poco relevante en las pesquerías debido a su baja representatividad en los reportes pesqueros frente a otras especies como el bocachico (P. magdalenae), que ha representado hasta el 50 \% de la pesquería en la cuenca del río
Magdalena (FAO, 2015). Esta baja representatividad se debe a las características morfológicas y estructurales del cuerpo de la arenca, tales como su forma ovalada y comprimida lateralmente, así como su gran número de espinas; sin embargo, algunas poblaciones de bajos ingresos económicos asentadas cerca de los ecosistemas donde habita la especie hacen uso de esta debido a su abundancia y facilidad de pesca (Olivero y Jhonson, 2002), así como por su sabor que resulta atractivo para los pobladores de la región. También se destaca su alto contenido de grasa que les permite a las personas freírlas sin utilizar otra fuente de aceite. Debido a estas razones, en algunas zonas del departamento de Bolívar (Colombia) ha recibido el calificativo de "Jacta pobre".

La importancia pesquera del T. magdalenae se ha evidenciado en estudios como el de Sánchez y Rueda (1999), en el delta del río Magdalena, donde se destaca a la especie como una de las dominantes en los complejos cenagosos de Pajarales y Salamanca, donde su captura por unidad de esfuerzo (CPUE) es de 0,9 y 0,89 g/lance, respectivamente. En Pajarales representa el $50 \%$ de la captura y en Salamanca el $67 \%$. En la CGSM LA CPUE fue apenas de $0.05 \mathrm{~g} /$ lance ( $8 \%$ de la captura).

La baja representación de la arenca en la pesquería de la CGSM también ha sido reportada en los informes nacionales de pesca y acuicultura de la Corporación Colombia Internacional durante el período 2007-2010 (CCI, 2007, 2008, 2009 y 2010), según los cuales el promedio mensual de desembarcos de $T$. magdalenae descendió significativamente después del año 2007 (figura 5a), principalmente durante el 2008, cuando se presentó el menor desembarco de la especie $(3,1 \mathrm{t})$.

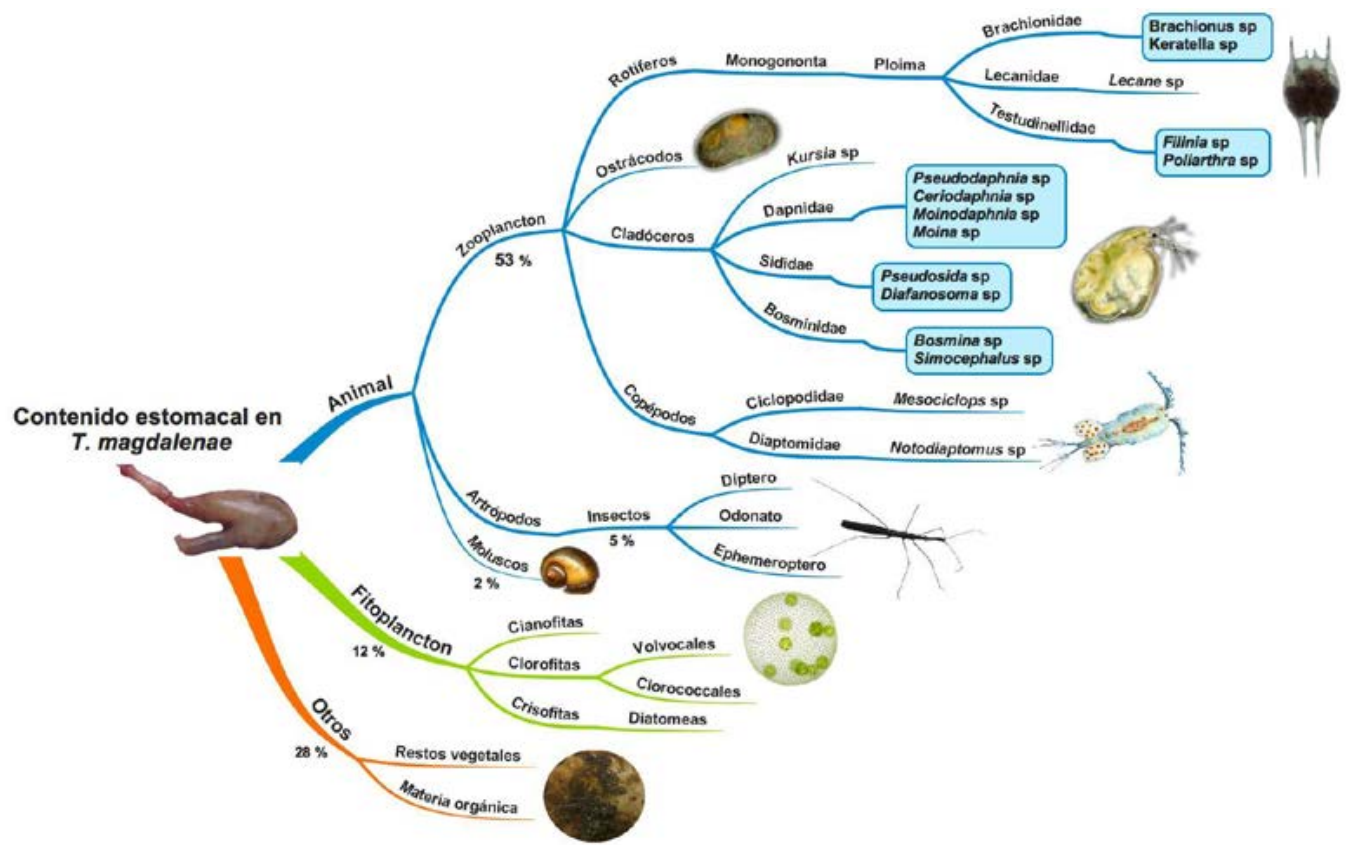

Figura 4. Principales representantes de la dieta de T. magdalenae. Los valores en las ramas indican la frecuencia relativa (\%) de los principales grupos taxonómicos. Modificado de Rodríguez y Rodríguez (1976), Benavidez y Valdelamar (2010) y Morales y García-Alzate (2018). 

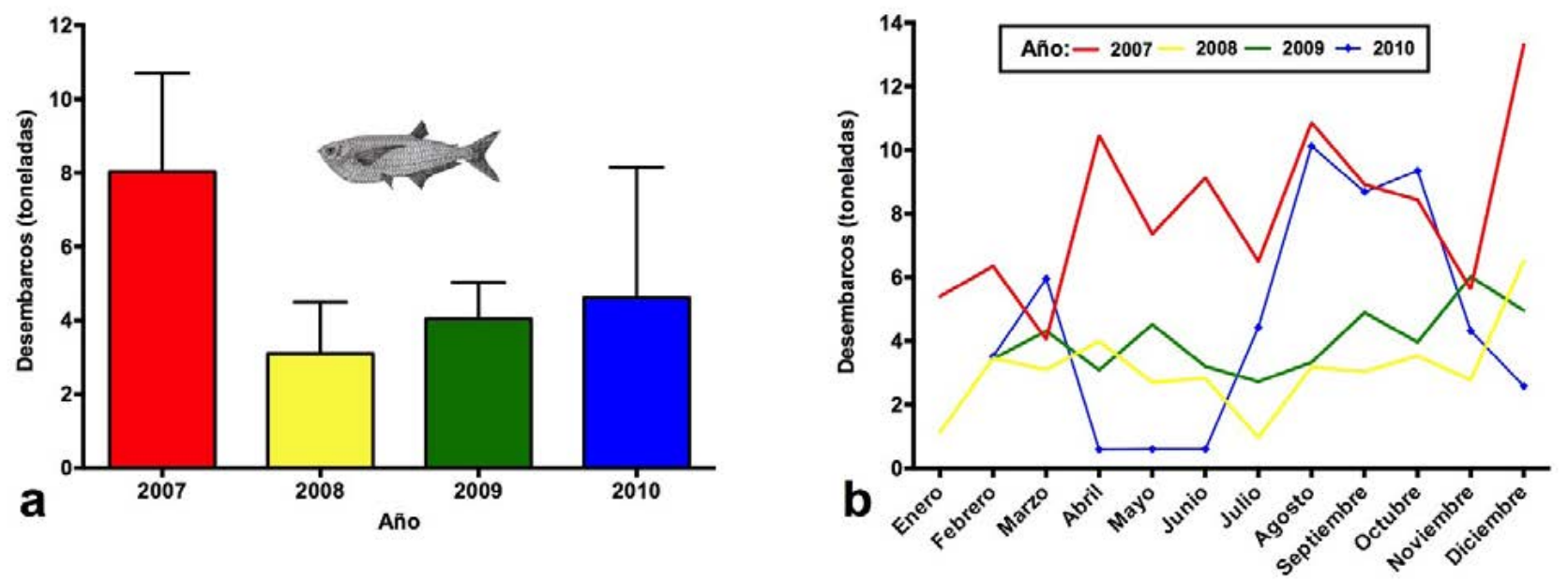

Figura 5. Comportamiento del promedio anual (a) y el total mensual (b) de desembarcos de T. magdalenae en la cuenca del río Magdalena y la Ciénaga Grande de Santa Marta, durante el periodo 2007 - 2010. Datos obtenidos de CCI (2007-2010).

El análisis de los mismos datos también permitió conocer que los mayores desembarcos de la especie se presentaron, casi siempre en los meses finales de cada año (figura $5 \mathrm{~b}$ ) que coinciden con la finalización del periodo lluvioso en la zona y con el inicio de la migración de la especie, factor que aumenta su captura, principalmente en los canales que comunican a este humedal con el río Magdalena.

La baja representatividad de T. magdalenae y otros peces tradicionales en los desembarcos pesqueros de la CGSM ha sido relacionada principalmente con la reorientación del esfuerzo de captura por parte de los pescadores hacia la mojarra lora (Oreochromis niloticus), la cual ofrece mayor rendimiento económico y de captura (CCI, 2009).

Otros estudios sugieren que $O$. niloticus posee alta eficiencia en la filtración del plancton y alto potencial reproductivo, lo que le permite aumentar rápidamente su población, estrategias que le confieren gran ventaja frente a las especies locales (Caraballo, 2009). Sin embargo, todavía faltan más estudios que permitan obtener resultados concluyentes sobre el grado de afectación que ha tendido T. magdalenae debido a la introducción de la mojarra Lora en los ecosistemas de las cuencas de los ríos Magdalena y Cauca.

Otro parámetro pesquero de interés es la talla media de madurez, la cual se ha reducido significativamente desde la década de los ochenta, cuando Escobar et al. (1983) registraron que variaba entre 19,5 y $20,9 \mathrm{~cm}$ longitud estándar (Le) para machos y hembras, respectivamente; en el año 2005, Bolívar et al. (2006a) reportaron tallas de madurez de 14,02 y 16,07 cm Le para machos y hembras, respectivamente. Sin embargo, es necesario realizar más estudios con el objetivo de actualizar esta información de gran utilidad para su manejo pesquero. A pesar de las anteriores consideraciones, T. magdalenae todavía conserva un estado poblacional estable según la lista roja de especies del IUCN (Jiménez-Segura y VillaNavarro, 2016). Igualmente, las fundaciones Humedales y Conservación de la Naturaleza (The Nature Conservancy et al. (2016) resaltan que durante el período comprendido entre 1977 y 2012 esta especie ha permanecido en el octavo puesto de la escala de abundancia, sin variación en el factor de cambio, y se mantiene la captura de especímenes con un promedio 17,1 cm Le, valor superior a la talla mínima legal $(15 \mathrm{~cm})$ reglamentada por la Autoridad Nacional de Pesca y Acuacultura (AUNAPUNIMAGDALENA,2013).

La reducción en la talla de madurez ocurre principalmente como respuesta a la presión por pesca a la que son sometidas algunas especies, como ha sido reportado en el caso de peces de otras latitudes como Macquaria novemaculeata y Sander lucioperca (de Roos et al., 2006; Lappalainen et al., 2016). Este comportamiento es preocupante debido a que en la actualidad no se dispone de información de base tecnológica para la reproducción artificial de la especie. Además, el único ensayo realizado sobre el cultivo de la especie mostró que su productividad no supera los $1000 \mathrm{~kg} /$ estanque (Aguilera, 2006). 


\section{Arenca como fuente de proteína y otros derivados para el consumo humano}

La tasa de consumo de T. magdalenae en el departamento de Bolívar es de 0,300 kg/día (De Miguel et al., 2014). Sin embargo, el consumo de este recurso tiene ciertas limitaciones asociadas a la dificultad de las poblaciones que habitan en zonas apartadas para evitar el rápido deterioro de su carne, ya que en la mayoría de los casos carecen de sistemas de refrigeración que funcionen de manera continua; sin embargo, en las investigaciones recientes sobre la conservación de la carne de esta especie recomiendan que los individuos sean salados con cloruro de sodio en pila seca o húmeda, para evitar su descomposición inmediata y, de esta manera, asegurar su consumo seguro (Acevedo et al., 2015; Tirado-Armesto et al., 2016).

La ingesta de arenca es importante para la dieta de muchas poblaciones debido a que sus músculos contienen entre 13,23 y 17,85 \% de proteína; igualmente, algunos ensayos efectuados para la obtención de concentrado proteico muscular de la especie indican que estos pueden llegar a contener entre 72 y $90 \%$ de proteína (Bolívar et al., 2007; Barrios y Quintana, 2012).

También se ha identificado que T. magdalenae posee en promedio 3,23\% de aceite crudo, en el cual predominan los ácidos grasos insaturados (56,77 \%) de la serie Omega 3 y 6 , donde el eicosapentanoico sobresale por su mayor abundancia, seguido del docosapentanoico, linolénico y el araquidónico, razón por la cual se ha considerado a la especie como una fuente potencial de materia prima para la fabricación de productos alimenticios como salsas, pastas para untar, aderezos productos farmacéuticos y/o medicinales (Padilla et al., 2012).

\section{Riesgos ambientales para la arenca}

La arenca ha estado sometida a múltiples tensores ambientales que ocasionaron la desaparición de sus poblaciones en ecosistemas como el río Bogotá que históricamente ha sido impactado de manera negativa por las actividades humanas (CAR-Conservación Internacional, 2012). Estas amenazas para la especie también se han mantenido vigentes en otros ecosistemas de la cuenca del río Magdalena, principalmente por alteraciones en las características fisicoquímicas del agua a causa de las actividades industriales, urbanísticas y mineras que vierten contaminantes químicos en éstos (Montes et al., 2013).

Entre los contaminantes vertidos a la cuenca del río Magdalena se destacan los de naturaleza química como hidrocarburos, plaguicidas y metales pesados (ManceraRodríguez y Alvarez-León, 2006; Tejeda-Benitez et al., 2016). Los reportes sobre el contenido de contaminantes en T. magdalenae datan de finales del siglo pasado, cuando Cala y Södergren (1999) registraron que los huevos de la especie tenían una concentración promedio de $7 \mathrm{ng} / \mathrm{g}$ del pesticida epóxido heptacloro.

En el mismo periodo se registraron los primeros reportes sobre el contenido en la especie de metales pesados como el mercurio (Olivero et al. 1997; Olivero y Solano, 1998). El ingreso del mercurio en los ecosistemas acuáticos de los departamentos de Bolívar, Antioquia, Caldas y Santander, donde también se ha reportado la presencia de T. magdalenae, ha ocurrido principalmente por causa de la minería aurífera; como consecuencia, se ha encontrado en la especie concentraciones que varían desde 0,02 hasta $0,39 \mu \mathrm{g} / \mathrm{g}$ de Hg-total, mientras que los pocos reportes de metil-mercurio indican que la concentración de este tóxico varía entre 0,03 y $0,06 \mu \mathrm{g} / \mathrm{g}$ (tabla 4).

Tabla 4. Valores de mercurio y metil-mercurio registrados para T. magdalenae. Periodo seco*; periodo lluvioso**

\begin{tabular}{|c|c|c|c|}
\hline Departamento & Hg-Total $(\mu \mathrm{g} / \mathrm{g})$ & Metil-Hg $(\mu \mathrm{g} / \mathrm{g})$ & Autor \\
\hline \multirow[t]{5}{*}{ Bolívar } & $0,29 \pm 0,06$ & & Olivero-Verbel et al. (2015) \\
\hline & $0,07 \pm 0,02$ & & Olivero y Johnson (2002) \\
\hline & ${ }^{* *} 0,39 \pm 0,07$ & & Olivero y Solano (1998) \\
\hline & ${ }^{*} 0,09 \pm 0,01$ & & Olivero y Solano (1998) \\
\hline & $0,10 \pm 0,06$ & & Olivero et al. (1997) \\
\hline Antioquia y Caldas & & $0,03-0,06$ & Álvarez et al. (2012a) \\
\hline Antioquia y Santander & $0,22 \pm 0,18$ & & Álvarez et al. (2012b) \\
\hline
\end{tabular}


La anterior situación resulta preocupante, debido a que una fracción importante del mercurio contenido en los especímenes de T. magadalenae está siendo consumido por poblaciones de escasos recursos económicos que viven principalmente en zonas afectadas por la actividad minera del oro, como el distrito minero de San Martín de Loba en el departamento de Bolívar, donde Olivero-Verbel et al. (2015) calcularon una tasa de ingesta diaria de Hg por comida de $0,86 \mu \mathrm{g} / \mathrm{kg} /$ día y un coeficiente de peligro alto $(8,58)$. Razón por la cual sugieren a los habitantes de la zona no consumir más de un espécimen a la semana, ya que el riesgo toxicológico es muy alto, especialmente para las mujeres embarazadas (Caballero, 2010).

En lo que respecta a la afectación de la arenca por contaminantes biológicos como bacterias y parásitos, son escasos los estudios que han enfocado este aspecto, entre los que se destaca el efectuado por Olivero et al. (2011) sobre parasitosis de esta y otras especies de peces en el Canal del Dique y la Bahía de Cartagena, en el Caribe colombiano; en dicho estudio los autores no encontraron parásitos en los especímenes de T. magdalenae, lo que sugiere que la especie tiene baja susceptibilidad a los parásitos presentes en esta zona. Sin embargo, todavía falta información que permita saber el estado en que se encuentra la especie en otros ecosistemas de la cuenca del río Magdalena-Cauca.

Además de los aspectos antes considerados, se deben tener en cuenta otros que ocurren a macroescala, como el cambio climático; las modelaciones efectuadas hasta la fecha sobre su impacto en la cuenca del río Magdalena indican que afectaría el comportamiento hidrológico del sistema, por la ocurrencia de lluvias y descargas de agua extremas (Jeuken et al., 2016). Aunque esta situación podría afectar a la arenca, cualquier aseveración que se pretenda hacer sobre ello sería especulativa, ya que actualmente no se dispone de estudios multi-temporales continuos que permitan tener detalle de parámetros básicos de la dinámica poblacional y del comportamiento ecofisiológico de la especie.

\section{Consideraciones finales}

Históricamente, T. magdalenae ha sido una especie invisibilizada en la literatura científica; sin embargo, esta especie es de gran importancia en la cuenca del río Magdalena debido al aporte que realiza a las pesquerías y como fuente de proteína para varias comunidades de escasos recursos económicos de la región; además posee un alto potencial de aplicación no solo en la industria alimenticia, sino también en la farmacéutica.
Los indicadores de pesca muestran que la arenca no se encuentra en peligro de sobre-explotación; sin embargo, es necesario realizar estudios multi-temporales enfocados al conocimiento de su dinámica poblacional y pesquera, lo cual será fundamental para desarrollar estrategias de manejo y conservación de la especie, sobre todo, ante el eventual impacto que tendría el cambio climático sobre la cuenca del río Magdalena, donde la especie podría sustituir a algunos de los principales recursos pesqueros que actualmente se encuentran en peligro de extinción.

\section{Agradecimientos}

A los miembros del Grupo de Química Ambiental y Computacional, por sus valiosos comentarios, y al Programa Nacional para Formación Doctoral (COLCIENCIASDoctorado Nacional No 647 de 2014).

\section{Referencias}

Aazami, J., Esmaili Sari, A., Abdoli, A., Sohrabi, H. y Van den Brink, P. 2015.Monitoring and assessment of water health quality in the Tajan, River, Iran using physicochemical, fish and macroinvertebrates indices. Environmental Management 56(1): 260-9.

Acevedo, D., Tirado, D. y Montero, P. 2015. Cinética de transferencia de masa durante salado de arenca (Triportheus magdalenae). Interciencia 40: 127-132.

Aguilera, M. 2006. El Canal del Dique y su subregión: Una economía basada en la riqueza hídrica. Editorial Banco de la República, Bogotá D.C.

Alonso, J., Escobar, F., Polo, C. y Puentes, V. 2014. Aguas Continentales: Cuenca del Magdalena. En: Puentes, V., Escobar, F. D., Polo, C. J. y Alonso, J., Editores. Estado de los Principales Recursos Pesqueros de Colombia - 2014. Generación del Conocimiento y la Información, Autoridad Nacional de Acuicultura y Pesca - AUNAP. Bogotá D.C.

Álvarez, S., Jessick, A., Palacio, J. y Kolok, A. 2012a. Methylmercury Concentrations in Six Fish Species from two Colombian Rivers. Bulletin of Environmental Contamination and Toxicology 88 (1): 65-68.

Álvarez, S., Kolok, A., Jiménez, L., Granados, C. y Palacio, J. 2012b. Mercury concentrations in muscle and liver tissue of fish from marshes along the Magdalena river, Colombia. Bulletin of Environmental Contamination and Toxicology 89(4): 836-840. 
Anaya, L., Ardila, C. y Carballo, P. 2016. Ictiofauna del Arroyo Grande de Corozal. Primer registro de 12 especies de peces para el departamento de Sucre, Colombia. Revista Colombiana Ciencia Animal 8 (Supl): 368-376.

Arango-Rojas, A., Jiménez-Segura, L. y Palacio-Baena, J. 2008. Variación espacio-temporal de la asociación de especies de peces en la Laguna Cahimbero, un humedal e la cuenca media del río Magdalena, Colombia. Actualidades Biológicas 30(89): 161-169.

Archila, S. 1993. Medio ambiente y arqueología en las tierras bajas del Caribe colombiano. Boletín Museo del Oro 34: 111164.

Ardila, C. 1994. Peces de agua dulce del departamento del Atlántico, Colombia. Dugandia 5(1): 3-12.

AUNAP-UNIMAGDALENA. 2013. Tallas mínimas de captura para el aprovechamiento sostenible de las principales especies de peces, crustáceos y moluscos comerciales de Colombia. Convenio 058 de 2013 entre la Autoridad nacional de acuicultura y pesca y la Universidad del Magdalena, Santa Marta.

Barrios, A. y García-Alzate, C. 2017. Influencia de las variaciones fisicoquímicas sobre cinco especies de peces de interés social y ambiental en el embalse el Guájaro, Atlántico, Colombia. Memorias del III Seminario Internacional en Ciencias Ambientales SUE-Caribe P: 63-66.

Barrios, F. y Quintana, S. 2012. Emulsiones alimentarias tipo aceite en agua estabilizadas con proteína de arenca (Triportheus magdalenae). Tesis de pregrado, Facultad de Ingeniería. Universidad de Cartagena, Cartagena, Colombia.

Benavidez, L. y Valdelamar, J. 2010. Contenido de Zooplancton estomacal en la arenca Triportheus magdalenae y su relación con la calidad de agua del embalse del Guájaro, departamento del Atlántico. Memorias del I Simposio Ambiental: Ecosistemas del Caribe colombiano 1:1-13.

Bolívar, F., Valdelamar, J., Bayuelo V. y Narváez, J. 2006a. Algunos aspectos reproductivos de la arenca, Triportheus magdalenae (Steindachner, 1878) en el Embalse del Guájaro y el Canal del Dique (Departamento del Atlántico). Memorias del III Congreso Nacional de acuacultura 3:92.

Bolívar, F., Valdelamar, J., Bayuelo, V. y Narváez J. 2006b. Estudio preliminar. Histología de ovarios y testículos de la arenca, Triportheus magdalenae (Steindachner, 1878) en el Embalse del Guájaro y el Canal del Dique (Departamento del Atlántico). Memorias del III Congreso Nacional de acuicultura 3:65.
Bolívar, F., Valdelamar, J., Bayuelo, V., Narváez, y Ortega E. 2007. Comportamiento de los lípidos hepáticos y de la proteína muscular durante el desarrollo gonadal de la arenca Triportheus magdalenae (Steindachner, 1878) (Pisces Characidae). Revista de la Facultad de Medicina Veterinaria $y$ de Zootecnia Suplemento 54(2): 240-241.

Caballero, L. 2010. Consumo de pescado y exposición al Metilmercurio. Riesgos y beneficios durante el embarazo. Revista Electrónica de Portales Médicos. http://www. portalesmedicos.com/publicaciones/articles/2207/1/ Consumo-de-pescado-y- exposicion-al-metilmercurioRiesgos-y-beneficios-durante-el-embarazo.html. Consultado: 6 de junio de 2017.

Cala, P. y Södergren, A. 1999. Ocurrence and distribution of organochlorine residues in fish from the Magdalena and Meta rivers in Colombia. Toxicological and Environmental Chemistry 71(1-2): 185-195.

Caraballo, P. 2009. Efecto de la tilapia Orechromis niloticus sobre la producción pesquera del embalse del Guájaro Atlántico - Colombia. Revista M.V.Z. Córdoba 14(3): 1796-1802.

CAR-Conservation International. 2012. Inventario de peces y crustáceos en tres fuentes hídricas de la jurisdicción de la CAR. Corporación Regional Autónoma de Cundinamarca Conservation International, Bogotá D.C.

Castellanos-Morales, C., Marino-Zamudio, L., Guerrero, L. y Maldonado-Ocampo, J. 2011. Peces del departamento de Santander, Colombia. Revista de la Academia Colombiana de Ciencias 35(135): 189-212.

Corporación Colombia Internacional (CCI). 2007. Pesca y acuicultura en Colombia. Corporación Colombia Internacional, Bogotá D.C.

Corporación Colombia Internacional (CCI). 2008. Pesca y Acuicultura en Colombia. Informe técnico regional, litoral Caribe y Pacífico. Corporación Colombia Internacional, Bogotá D.C.

Corporación Colombia Internacional (CCI). 2009. Pesca y Acuicultura en Colombia. Informe técnico regional, litoral Caribe y Pacífico. Corporación Colombia Internacional, Bogotá D.C.

Corporación Colombia Internacional (CCI). 2010. Pesca y acuicultura en Colombia. Corporación Colombia Internacional, Bogotá D.C.

Dahl, G. 1971. Los peces del norte de Colombia. Ministerio de Agricultura. INDERENA, Bogotá D.C. 
De Miguel, E., Clavijo, D., Ortega M. y Gómez A. 2014. Probabilistic meta-analysis of risk from the exposure to $\mathrm{Hg}$ in artisanal gold mining communities in Colombia. Chemosphere 108: $183-189$.

De Roos, A., Boukal, D. y Persson, L. 2006. Evolutionary regime shifts in age and size at maturation of exploted fish stock. Proceedings of the Royal Society of London. Series $B$ Biological Sciences 273: 1873-1880.

Escobar, J., M. Zárate, M. Valderrama, C. Lara y Fonseca, C. 1983. Tallas mínimas y medias de maduración para catorce especies ícticas de interés comercial de la cuenca Magdalena. Inderena. Revista Divulgación Pesquera 21(2-3): 1- 42.

Food and Agriculture Organization of the United Nations (FAO). 2015. Colombia. Pesca en cifras-/2014. FAO, Minagricultura, Bogotá D.C.

Fernández, M., Martínez, A. y Molina, A. 2008. Propuesta del plan de manejo de la ciénaga de San Antonio, localizada en los municipios de cerro de San Antonio y Concordia, departamento del Magdalena. Tesis de Magíster, Pontificia Universidad Javeriana, Bogotá, Colombia.

Gil-Calderón, D. y Márquez, E. 2015. Diseño y evaluación de cebadores de Novo para la amplificación de microsatélites en Triportheus magdalenae. XIII Congreso colombiano de Ictiología Amazonas. IV Encuentro de Ictiólogos suramericanos. Leticia, Amazonas. Asociación Colombiana de Ictiólogos 58-59.

Gutiérrez, C. y Pinilla, A. 2016. Efectos de la conectividad local sobre los ensambles de peces en una planicie de inundación tropical. Caldasia 38(2): 300-313.

Hernández, F. 2015. Pesca y organización comunitaria frente a un recurso en declive. Caso Ciénaga de María la Baja, Bolívar. Universidad Javeriana, Corporación Desarrollo Solidario y Observatorio de territorios étnicos y campesinos 1-19 Disponible en: http://etnoterritorios.org/apc-aa-il es/92335f7b3cf47708a7c984a309402be7/cartilla-parapescadoresfrancyh.pdf Consultado: 6 de junio de 2017

Holmlund, C. y Hammer, M. 1999. Ecosystem services generated by fish populations. Ecological Economics 29: 253-268.

Jeuken, A., Bouaziz, L., Corzo, G. y Alfonso, L. 2016. Analyzing needs for climate change adaptation in the Magdalena River basin in Colombia. En: Leal Filho, W., Musa, H. Cavan, G., O'Hare P. y Seixas, J., Editor. Climate change adaptation, resilience and hazards. Cham: Springer 163
Jiménez-Segura, L., López-Casa, S., Morales-Betancourt, M. y González-Cañon, G. 2011. Triportheus magdalenae. Catálogo de especies. Familia Characiformes. Capítulo 7. En: Lasso C., Agudelo E., Jiménes-Segura F., Ramírez-Gil, H., Morales Betancourt M., Ajíaco-Martínez R., de Paula F., Usma J., Muñoz S. y Sanabria, A., Editores. Catálogo de los recursos pesqueros continentales de Colombia. Serie Editorial Recursos Hidrobiológicos y Pesqueros Continentales de Colombia. Instituto de Investigación de los Recursos Biológicos Alexander von Humboldt (IAvH), Bogotá D.C.

Jiménez-Segura, L. y Villa-Navarro, F. 2016. Triportheus magdalenae. The IUCN Red List of Threatened Species 2016:e.T49830754A61474148.http://dx.doi.org/10.2305/ IUCN.UK.2016-1.RLTS.T49830754A61474148. Consultado: 6 de junio de 2018.

Lahnsteiner, F. y Mansour, N. 2012. The effect of temperature on sperm motility and enzymatic activity in brown trout Salmo trutta, burbot Lota lota and grayling Thymallus thymallus. Journal of Fish Biology 81(1): 197-209.

Lappalainen, A., Saks, L., Sustar, M., Heikinheimo, O., Jurgens, K., Kokkonen, E., Kurkilahti, M., Verliin, A. y Vetemaa, M. 2016. Length maturity as potential indicator of fishing pressure effects on coastal pikkeperch (Sander lucioperca) a stocks in the northern Baltic Sea. Fisheries Research 174: 47-57.

López-Casas, S. y Jiménez-Segura, L. 2015. Especies potádromas del Magdalena: Listado actualizado, distancias, recorridos y velocidades. XIII Congreso colombiano de Ictiología Amazonas. IV Encuentro de Ictiólogos suramericanos. Leticia, Amazonas. Asociación Colombiana de Ictiólogos 13: 164.

Malabarba, M. 2004. Revision of the Neotropical genus Triportheus Cope, 1872 (Characiformes: Characidae). Neotropical Ichthyology 2(4):167-204.

Maldonado-Ocampo, J. y Usma-Oviedo, J. 2006. Estado del conocimiento sobre peces dulceacuícolas en Colombia. Tomo II. En: Chaves, M.E. y Santamaría, M., Editores. 2006. Informe Nacional sobre el avance en el conocimiento y la información de la biodiversidad 1998-2004. Instituto de Investigación en Recursos Biológicos Alexander von Humboldt, Bogotá D.C.

Mancera-Rodríguez, N. y Álvarez-León, R. 2006. Estado del conocimiento de las concentraciones de mercurio y otros metales pesados en peces dulceacuícolas de Colombia. Acta Biológica Colombiana 11(1): 3-23. 
Mariguela, T., Roxo, F., Foresti, F. y Oliveira, C. 2015. Phylogeny and biogeography of Triportheidae (Teleostei: Characiformes) based on molecular data. Molecular Phylogenetics and Evolution 96: 130-139.

Marquioni, V., Bertollo, L., Diniz, D. y de Bello, M. 2013. Comparative chromosomal mapping in Triportheus fish species. Analysis of synteny between ribosomal genes. Micron 45: 129-135.

Mojica, J. 2002. Las pesquerías de la cuenca del río Magdalena: ejemplo a no repetir. En: Mojica, J.I., Castellanos, C., Usma J.S. y Álvarez, R. Editores. Libro rojo de peces dulceacuícolas de Colombia. Instituto de Ciencias Naturales. Instituto Von Humboldt y Ministerio del Medio Ambiente, Bogotá.

Mojica, J., Galvis, G., Sánchez-Duarte, P., Castellanos, C. y Villa-Navarro, F. 2006. Peces del valle medio del río Magdalena, Colombia. Biota Colombiana 7(1): 23-37.

Montes, R., Navarro, I., Domínguez, R. y Jiménez, B. 2013. Modificación de la capacidad de autodepuración del río Magdalena ante el cambio climático. Tecnología y ciencias del agua 4(5): 71-83.

Morales, J. y García-Alzate, C. 2018. Ecología trófica y rasgos ecomorfológicos del pez Triportheus magdalenae (Characiformes: Triportheidae) en el embalse del Guájaro, río Magdalena, Colombia. Revista de Biología Tropical 66(3): 1208-1222.

Moreno, G., 2005. Ecología básica de Triportheus magdalenae y Pimelodus clarias en la ciénaga de Ayapel, (cuenca media del río San Jorge) durante el ciclo hidrológico 2004-2005. Tesis de pregrado, Instituto de Biología, Universidad de Antioquia, Medellín, Colombia.

Motta, N., Della-Fina, N., Souza, C., Rodrigues, E. y Amorim, A. 2016. Analysis of food habits of skate Rioraja agassizii (Elasmobranchii, Rajidae) from southern Brazil. Brazilian Journal of Biology 76(2): 469-475.

Nakamura, M. 2013. Morphological and Physiological Studies on Gonadal Sex Differentiation in Teleost Fish. AquaBioScience Monographs 6(1): 1-47.

Ochoa-Orrego, L.E., Jiménez, L.F., y Palacio, J. 2015. Ictioplancton en la Ciénaga de Ayapel, río San Jorge (Colombia): cambios espacio temporales. Boletín científico del Centro de Museos de la Universidad de Caldasia 19 (1): 103-114.

Olivero, J. Caballero-Gallardo, K., Arroyo-Salgado, B. 2011. Nematode infection in fish from Cartagena Bay, North of Colombia. Veterinary Parasitology 177: 119-126.
Olivero, J. y Johnson, B. 2002. El lado gris de la minería del Oro. La contaminación con mercurio en el norte de Colombia. Universidad de Cartagena - Editorial Universitaria, Cartagena.

Olivero, J., Navas, V. Pérez, A., Solano, B., Acosta, I., Argüello, E. y Salas, R. 1997. Mercury levels in muscle of some fish species from the Dique Channel. Bulletin of Environmental Contamination and Toxicology 58: 865-870.

Olivero, J. y Solano, B. 1998. Mercury in environmental samples from a waterbody contaminated by gold mining in Colombia, South America. Science of the Total Environmental 217: 83-89.

Olivero-Verbel, J., Caballero-Gallardo, K., y Turizo-Tapia, A. 2015. Mercury in the gold mining district of San Martin de Loba, South of Bolivar (Colombia). Environmental Science and Pollution Research 22(8): 5895-5907.

Ortíz-Muñoz, V. y Alvarez-León, R. 2008. Caracterización de la tolerancia ambiental de las comunidades ícticas en subsidiarios de los ríos Cauca y Magdalena, Colombia. Memoria de la Fundación La Salle de Ciencias Naturales 169: 7-20.

Padilla, D., Beleño, L. y Cuello, R. 2012. Evaluación de la extracción del aceite de la Triportheus magdalenae y análisis del perfil lipídico del aceite crudo. Revista Iberoamercicana para la investigación y el Desarrollo Educativo 3(5): 1-19.

Peña-León, G. 2011. Pescadores de los raudales del río Magdalena durante el periodo formativo tardío. Caldasia 33(2): 295-314.

Peña, G. 2013. Pescadores de los raudales del río Magdalena durante el período Formativo Tardío (siglos $V$ al I a.C.). Universidad Nacional de Colombia, Bogotá.

Polanía, J., Santos-Martínez, A., Mancera-Pineda, J. y Arboleda, L. 2001. The Coastal Lagoon Ciénaga Grande de Santa Marta, Colombia. En: Seeliger, U. y Kjerfve, B., Editor. Coastal Marine Ecosystems of Latin. Springer, BerlínHeidelberg.

Rodríguez, G. y Rodríguez, C. 1976. Contribución al estudio bioecológico de la arenca (Triportheus magdalenae, Steindachner, 1878). INDERENA, Medellín.

Sánchez, C. y Rueda, M. 1999. Variación de la diversidad y abundancia de especies ícticas dominantes en el delta del río Magdalena, Colombia. Revista Biología Tropical 47(4): 1067 - 1079. 
Smith, J., Baumgartner, L., Suthers, I., Taylor, M. 2011. Generalist niche, specialist strategy: the diet of an Australian percichthyid. Journal of Fish Biology 78: 1183-1199.

Tejeda-Benitez, L., Flegal, R., Odigie, K. y Olivero-Verbel, J. 2016. Pollution by metals and toxicity assessment using Caenorhabditis elegans in sediments from the Magdalena River, Colombia. Environmental Pollution 212: 238-250.

Tirado-Armesto, D., Acevedo-Correa, D. y Montero-Castillo, P. 2016. Secado de arenca (Triportheus magdalenae): Influencia del salado en pila seca y humeda. Biotecnología del Sector Agropecuario y Agroindustrial 14(2): 57-64.

The Nature Conservancy, Fundación Alma, Fundación Humedales, AUNAP. 2016. Estado de las planicies inundables y el recurso pesquero en la macrocuenca Magdalena-Cauca y propuesta para su manejo integrado. The Nature Conservancy, Bogotá.

Vargas-Chacoff, L., Saavedra, E., Oyarzún, R., MartínezMontaño, E., Pontigo, J.P., Yañez, Ruiz, A., Jarabo, I., Mancera, J.M., Ortíz, E. y Bertrán, C. 2015. Effects on the metabolism, growth, digestive capacity and osmoregulation of juvenile of Sub-Antarctic Notothenioid fish Eleginops maclovinus acclimated at different salinities. Fish Physiology Biochemichal 41(6): 1369-81.

Villa-Navarro, F., García-Melo, J., García-Melo, N., BriñezVasques, F. y P. Zúñiga-Upegui. 2003. Biodiversidad Faunística de la cuenca del río Coello - Biodiversidad Regional Fase I. Tomo II. Informe presentado a Cortolima. Universidad del Tolima, Ibagué.

Villa-Navarro, F., Zuñiga-Upegui, P., Castro-Roa, D., GarcíaMelo, J. García-Melo, L. y Herrada-Yara, M. 2006. Peces del alto Magdalena, cuenca del río Magdalena, Colombia. Biota Colombiana 7(1): 3-21.

Zapata, L. y Usma J. 2013. Guía de las especies Migratorias de la Biodiversidad en Colombia. Peces. Vol. 2. Ministerio de Ambiente y Desarrollo Sostenible / WWF-Colombia. Bogotá, D.C.

Zubiria, W., Lacayo, A., Acero, A. y Narváez, J. 2008. Diversidad y abundancia de la ictíofauna de un complejo de Lagunas costeras en una reserva natural del Caribe colombiano. Memoria de la Fundación La Salle de Ciencias Naturales 170: 125-139.

Citar como: Valdelamar-Villegas J.C. 2018. Apuntes sobre la importancia ecológica, ambiental y social de la arenca Triportheus magdalenae (Steindachner, 1878). Un ejemplo de endemismo invisibilizado. Intropica 13(2): 152-165 DOI: http://dx.doi.org/10.21676/23897864.2628. 